ISASE 2020

\title{
Optimization Model to Express Emotions of Pet Robots
}

\author{
Chihiro MORITA* and Masataka TOKUMARU ** \\ * Graduate School of Kansai University, 3-3-35 Yamate-cho, Suita-shi, Osaka 564-8680, Japan \\ ** Kansai University, 3-3-35 Yamate-cho, Suita-shi, Osaka 564-8680, Japan
}

\begin{abstract}
In this study, we propose an emotional expression optimization model that is focused on action. Humanoid robots can express emotions through speech or gestures. However, most pet robots do not have the ability to speak like humans; thus, it is necessary for them to express emotions only through various actions. To resolve this issue, we apply an interactive tabu search, which was proposed in our previous emotion generation model. According to the proposed model, robots can change their actions depending on the intended emotional expressions, thus, they can appropriately express complex emotions. As a result of experimental verification, the proposed model is effective at searching for users preferred emotional expression actions.
\end{abstract}

Keywords: Pet Robots, Emotion Expression, Optimization Model, Interactive Tabu Search

\section{INTRODUCTION}

In recent years, communication robots incorporating artificial intelligence have become increasingly common. In particular, pet robots are designed to provide emotional communication through actions. Therefore, pet robots need to be sensitive to emotions and to demonstrate various reactions in return, like a living creature.

However, these types of robots have a problem that people get tired of them quickly, because the algorithms implemented in such robots are simple and have a limited variety of emotions and expressions. To resolve this problem, two types of algorithms are required. One type is an emotion generation algorithm that is used to facilitate complex emotions. The other type is an optimization algorithm that changes actions to express the generated emotions accordingly.

In our previous studies, for emotion generation algorithms, we proposed the emotion generation model that was based on emotional psychology [1,2]. In these studies, a robot was implemented to generate complex emotions like a living creature. Moreover, regarding the optimization of emotional expressions, the result of previous studies dedicated to emotion expressions of robots demonstrated that appropriate emotional expressions of robots affected human social decisionmaking and emotional experience $[3,4]$. Therefore, a method to enable robots to express appropriate emotions was proposed [5].

Considering that the emotion generation model proposed in the previous study is assumed to be used in a humanoid partner robot, such robot can use speech and gestures to express emotions. However, many pet robots are intended to imitate existing or nonexistent animal shapes, and consequently, their communication method is based on the animal model. Therefore, it is necessary for pet robots to express emotions using only actions.

In this study, we propose an emotional expression optimization model that is focused on actions. We apply the interactive tabu search proposed in our previous study. According to the proposed model, robots can change their actions according to emotional expressions; thus, they are able to express complex emotions appropriately.

\section{PROPOSED MODEL}

\subsection{Structure of the proposed model}

Compared to the previous emotion generation model [2], the model proposed in this study enhanced the optimization of emotional expressions. Figure1 shows the structure of the proposed model.

First, a robot generates its emotion on the basis of users external input and express the emotion by a corresponding

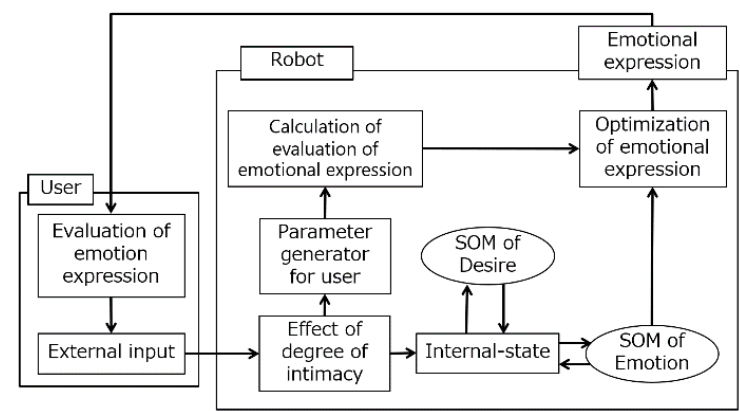

Figure 1: Structure of the proposed model 
action. Next, the model estimates the user evaluation of robot's emotional expressions. The model optimizes the emotional expressions based on the estimated evaluation. In this study, the interactive tabu search is used as the optimization method. The proposed model enables a robot to optimize emotional expressions on the basis of user's subjective evaluation.

\subsection{Interactive tabu search}

The interactive tabu search is an optimization method that is based on the subjective evaluation of the user. In this method, users evaluate solutions according to their sensitivity to reflect the evaluation results in the succeeding solutions.

First, an initial individual is randomly generated and presented to the user. Second, the user is expected to evaluate the provided individual on the basis of subjective evaluation and then to select the most favorite individuals among the provided ones. Simultaneously, the selected individual is stored in the tabu list. Neighborhood solution individuals are generated on the basis of the selected individual and then are presented to the user. At this point, the generated individuals differ from those included in the tabu list in terms of the bit sequence. These processes are repeated until the end condition determined by the user is satisfied. Finally, the individuals in the tabu list are presented to and evaluated by the users.

\subsection{Estimation of the user evaluation of emotional expressions}

In this study, the target to be optimized is the set of emotional expression actions performed by the robot. To accurately obtain the user evaluation, they need to input the evaluation or selection results into the robot. However, in the natural communication between the pet robot and the user, it may be difficult for the user to input their evaluations that corresponded to the emotional expression actions every time the robot performs an action. Therefore, for robots to optimize their emotional expression actions in natural communication, robots need to predict whether emotions have been correctly transmitted to users and whether the user likes the action based on the external input provided by them.

In this study, the robot attempts to obtain the users external input that eliminates the desire by the emotional expression action to express to the user its desire. Therefore, it can be considered that the robot is able to express emotions appropriately when the robot's desire has been eliminated by the user. To incorporate the values related to desires, in the previous studies, the degrees of
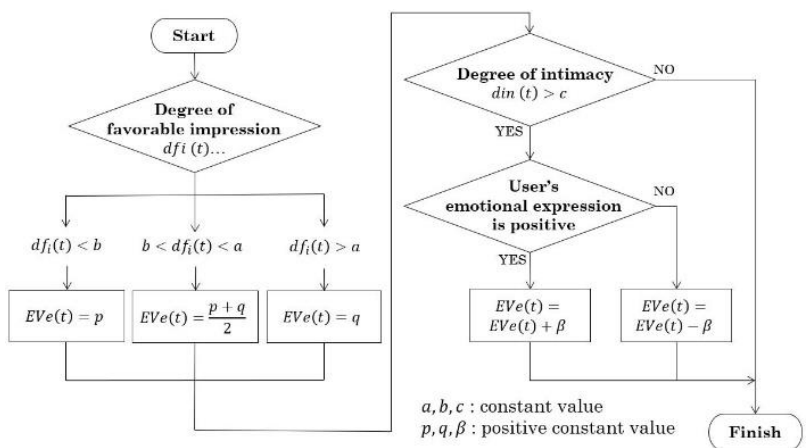

Figure 2: Flowchart of calculation process to obtain the user evaluation value for the emotional expression action

the favorable impression and intimacy have been calculated on the basis of the evaluation value corresponding to the state of desire.

Therefore, in this study, user evaluation with regard to the emotional expression of the robot is estimated on basis of the degrees of favorable impression and intimacy. Figure 2 shows a flowchart for calculating the process of the user evaluation value corresponding to the emotional expression action. When the value of the degree of the favorable impression $d f i(t)$ is larger than the constant value $a$, it means that the desire of the robot is appropriately eliminated by the user. Thus, it is assumed that the emotion expression has been also performed appropriately; therefore, the evaluation value corresponding to the emotional expression action is a positive constant value $p$. When the value of the degree of intimacy $\operatorname{din}(t)$ is larger than the constant value $c$, it is considered that the robot regularly and correctly expresses emotions to the user. Therefore, as the robot searches for an emotional expression action that is the most preferable by the user, the evaluation value is modified according to the user's emotional expression. It is assumed that the user selects the individual with the highest evaluation value for the emotional expression action.

\section{EXPERIMENT}

\subsection{Experiment outline}

In this experiment, the effectiveness of the proposed model is verified by implementing the proposed model and the comparison model in the robots and comparing them. The comparison model executes only a single emotional expression action corresponding to the emotion. The subjects were 8 university students. We employed EZ-Robot SIX Hexapod from EZ-Robot Inc., which is shown in Figure 3.

First, the user sits in a chair and faces one robot in front. The user is not notified which robot is implemented with 


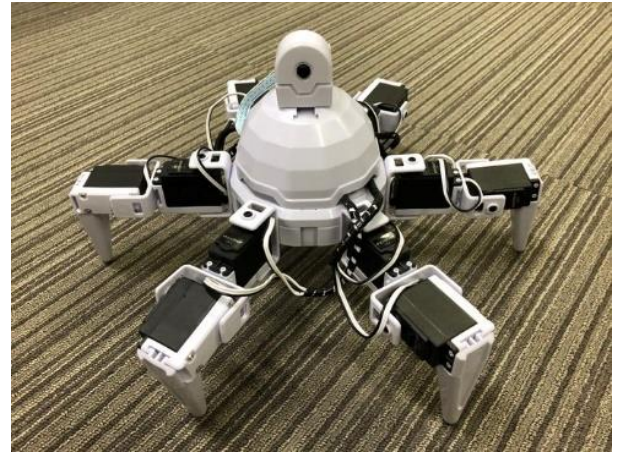

Figure 3: Robot implemented in the proposed model

the proposed model. Next, we explain to the user that there are 4 types of selectable actions when communicating with the robot: "feeding", "calling out", "playing", and "ignoring". The user tries to communicate with the robot by making an action from these 4 types. The robot changes after 100 interactions. At the point of interaction with each robot 400 times, a total of 800 times, the user answers a four-choice questionnaire to investigate the impression of each robot. Table 1 shows the questionnaire items.

Table 2 shows the common parameters used in the experiment. First, the elements of the internal state changed from the previous research are shown in Table 2(a). Next, Table 2(b) shows the common parameters of emotion generation. Finally, Table 2(c) shows the types of external-stimulation. Table 3 shows the parameters used for optimizing the emotion expression of the proposed model.

Table 1: Questionnaire items

\begin{tabular}{|c|c|}
\hline Question Number & Contents \\
\hline 1 & $\begin{array}{c}\text { For which robot did you understand its } \\
\text { emotion from the movement? }\end{array}$ \\
\hline 2 & $\begin{array}{c}\text { Which robot did you prefer in terms of its } \\
\text { emotional expression? }\end{array}$ \\
\hline
\end{tabular}

Table 2: Common parameters

(a) Elements of the internal state changed from the previous research

\begin{tabular}{|c|c|c|}
\hline Factor of internal-state & \multicolumn{2}{|c|}{ Category } \\
\cline { 1 - 2 } Hunger & \multicolumn{2}{|c|}{ States influenced by desires } \\
\cline { 1 - 1 } Comfort & \multicolumn{2}{|c|}{} \\
\hline Growth & Physiological need & \multirow{2}{*}{$\begin{array}{c}\text { Container } \\
\text { for desires }\end{array}$} \\
\cline { 1 - 2 } Love & $\begin{array}{c}\text { Love and } \\
\text { belonging }\end{array}$ & \\
\hline Appetite & Esteem & \\
\hline A desire of Belonging & & \\
\hline A desire of Attention & &
\end{tabular}

(b) Common parameters

\begin{tabular}{|c|c|}
\hline \multicolumn{2}{|c|}{ Common parameters } \\
\hline Number of times of learning & 400 \\
\hline Number of times of external-input & $-1.0-1.0$ \\
\hline \multicolumn{2}{|c|}{ Parameter of emotion generation SOM } \\
\hline Combination load & $-1.0-1.0$ \\
\hline Input layer neuron & 22 \\
\hline Output layer neuron & Individual value 3, \\
\hline Max 9
\end{tabular}

(c)Types of external stimulation

\begin{tabular}{|c|c|}
\hline Type of external-stimulation & Category \\
\hline Feeding & Stimulation by users \\
\hline Calling & \\
\hline Playing & Stimulation by \\
\hline Ignoring & action \\
\hline $\begin{array}{c}\text { Emotional expression } \\
\text { (Desire has occurred) }\end{array}$ \\
\hline $\begin{array}{c}\text { Emotional expression } \\
\text { (Desire does not have occurred) }\end{array}$ & \\
\hline
\end{tabular}

Table 3: Parameters used for optimizing the emotion expression of the proposed model

\begin{tabular}{|c|c|}
\hline \multicolumn{2}{|c|}{ Parameters of the tabu search } \\
\hline Number of times of learning & 400 \\
\hline $\begin{array}{c}\text { Number of neighborhood } \\
\text { solutions }\end{array}$ & 4 \\
\hline Taboo list length & 4 \\
\hline Parameter of the estimation of the user evaluation \\
\hline$a$ & 0.1 \\
\hline$b$ & -0.1 \\
\hline$c$ & 0.4 \\
\hline$p$ & 0.6 \\
\hline$q$ & 0.2 \\
\hline$\beta$ & 0.2 \\
\hline
\end{tabular}

\subsection{Results and discussions}

Figure 4 shows the results of the questionnaire. Figure 5 shows the transition of the average degree of intimacy.

We consider whether a change in the emotional expression action was appropriate for the user. The emotional expression appropriate for the user is an expression in which the emotion of the robot is transmitted to the user and the expressional action is favorable for the 


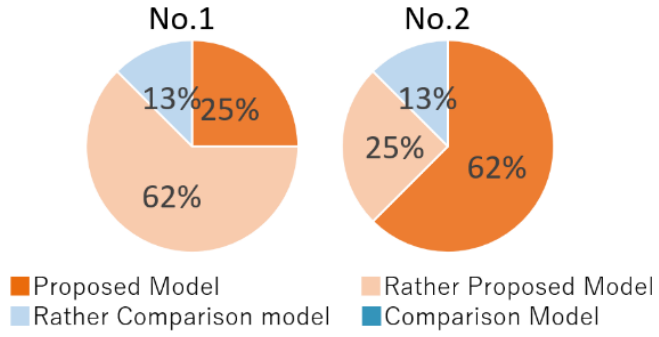

Figure 4: Questionnaire results

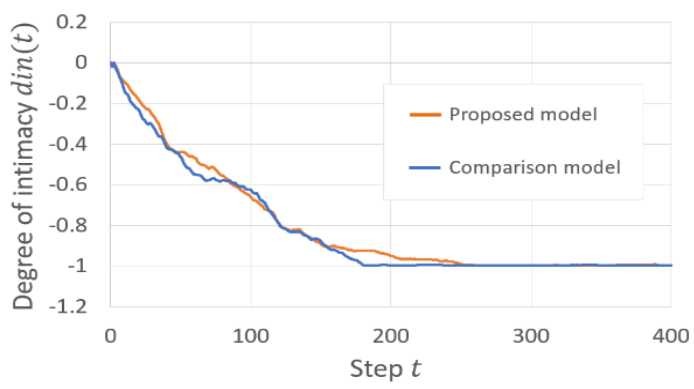

Figure 5: Average of the transition of the degree of intimacy

user. Figure 4 confirmed that $62 \%$ of the users answered that they could understand the emotion from the proposed model's actions compared with that of the comparison model. Therefore, it is difficult for users to clearly understand the emotion expressed by the proposed model. However, it is considered that the user clearly feels that the robot changes the expression behavior based on their own emotion. It was also confirmed that $62 \%$ of the users answered that the proposed model was able to make more favorable emotional expressions. Therefore, it is considered that the proposed model was able to take the emotional expression action that the user liked.

Next, we consider whether a change in the emotional expression behavior was appropriate for the robot. The emotional expression action appropriate for the robot is one that prompts the user to eliminate the desires. Figure 5 confirmed that the degree of intimacy continued to decrease in both the proposed and comparison models. Therefore, it is observed that the robot was not able to obtain external stimulation from the user that could eliminate its own desires. This suggests that the proposed model could not properly change the emotion expression for the robot.

This occurred because the method of generating or searching for emotional expression action was not appropriate. In this experiment, it was necessary to search for a solution within a limited experimental period. Thus, we set 9 basic actions and limited the solution space. Therefore, it is considered that it was not possible to search for the emotional expression action that could correctly convey the emotion to the user.

\section{CONCLUSION}

In this study, to enable a more comfortable communication between humans and pet robots, we proposed the optimization model to define actions of a pet robot according to the emotional expressions. To verify the applicability of the proposed model, we implemented the method in a robot and performed a comparative experiment. The results suggest that the proposed model can optimize emotional expression action in terms of whether it is favorable for users. However, it was considered that the emotional expression action cannot be optimized in terms of whether the robots emotions could be correctly conveyed to the user. In the future, it is necessary to carry out experiments by assuming longer-term communication, and to consider the coding method of actions.

\section{REFFERENCES}

[1] H. Nagano, M. Harata, M. Tokumaru, "Developing sophisticated robot reactions by long-term human interaction", the 15th International Conference on Human-Computer Interaction: Human-Computer Interaction, Towards Intelligent and Implicit Interaction, Lecture Notes in Computer Science Volume 8008, pp.319-328, 2013.

[2] S. Kinoshita, H. Takenouchi, M. Tokumaru, "An Emotion Generation Model for a Robot that Reacts after Considering the Dialogist", $7^{\text {th }}$ IEEE International Conference Humanoid Nanotechnology Information Technology Communication and Control Environment and Management (HNICEM2014) IS-09, pp.1-6, 2014.

[3] D. Leyzberg, E. Avrunin, J. Liu, B. Scassellati, "Robots that express emotion elicit better human teaching", 6th ACM/IEEE International Conference on Human-Robot Interaction (HRI), IEEE, pp. 347-354, 2011.

[4] C. L. Bethel, R. R. Murphy, "Survey of non-facial/nonverbal affective expressions for appearanceconstrained robots", IEEE Transactions on Systems, Man, and Cybernetics, Part C (Applications and Reviews), pp.83-92, 2005.

[5] T. Tsujimoto, Y. Takahashi, S. Takeuchi, "RNN with Russell's circumplex model for emotion estimation and emotional gesture generation", 2016 IEEE Congress on Evolutionary Computation (CEC). IEEE, pp.1427-1431, 2016. 